\title{
Development of the Dual Aerodynamic Nozzle Model for the NTF Semi-Span Model Support System
}

\author{
Gregory S. Jones ${ }^{1}$, William E. Milholen, $\mathrm{II}^{2}$, and Scott L. Goodliff ${ }^{3}$ \\ NASA Langley Research Center, Hampton, VA, 23681
}

The recent addition of a dual flow air delivery system to the NASA Langley National Transonic Facility was experimentally validated with a Dual Aerodynamic Nozzle semi-span model. This model utilized two Stratford calibration nozzles to characterize the weight flow system of the air delivery system. The weight flow boundaries for the air delivery system were identified at mildly cryogenic conditions to be 0.1 to $23 \mathrm{lbm} / \mathrm{sec}$ for the high flow leg and 0.1 to $9 \mathrm{lbm} / \mathrm{sec}$ for the low flow leg. Results from this test verified system performance and identified problems with the weight-flow metering system that required the vortex flow meters to be replaced at the end of the test.

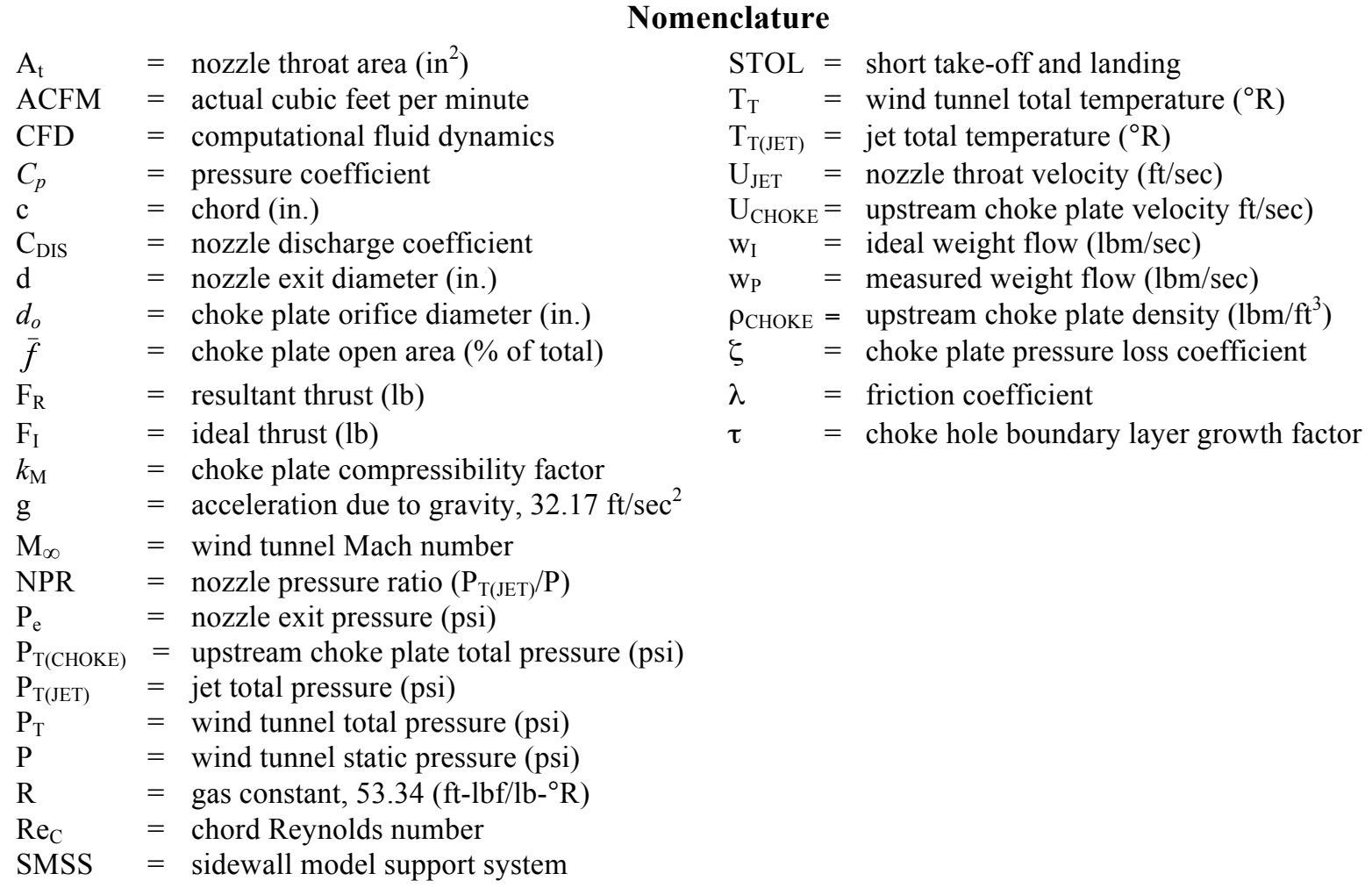

\footnotetext{
${ }^{1}$ Research Engineer, Flow Physics and Control Branch, Mail Stop 170, AIAA Senior Member

${ }^{2}$ Research Engineer, Configuration Aerodynamics Branch, Mail Stop 499, AIAA Senior Member

${ }^{3}$ Test Engineer, Jacobs Technology, Inc., Mail Stop 267, AIAA Senior Member
} 


\section{Introduction}

$\mathrm{R}$ ecently, the NASA Langley's National Transonic Facility (NTF) was upgraded with a flow control system to expand the capabilities of this high Reynolds number wind tunnel to include propulsion and advanced active flow technologies. ${ }^{1}$ In this paper we focus on the development and testing of a dual-flow nozzle model which serves as a reference model for blowing configurations at the NTF and was used to test the limits of the new blowing system.

Many of the advanced aircraft being designed today utilize advanced propulsion and active flow control systems. ${ }^{2}$ Energy efficiency, community noise, runway independence, and cruise efficiency can no longer be optimized independently because of the close coupling of the engine, airframe, and wing. It has also become more apparent in trade studies that high lift and cruise are not mutually exclusive with regard to performance optimization.. The successful flights of several short takeoff and landing (STOL) technology demonstrators proved that transport aircraft could achieve short field lengths; however, these aircraft were not optimized for transonic cruise. The YC-14 and YC-15, which employed upper surface blowing and externally blown flaps, were limited to cruise Mach numbers less than 0.7. These limitations were attributed to the propulsion integration, thick airfoils, low wing sweep, and aft end drag typical of these transports. ${ }^{3}$ In the present day, these inefficiencies can no longer be tolerated and advances in active flow control and computational fluid dynamics (CFD) schemes are expected to enhance performance and optimization efforts.

While great strides have been made in computations being used in the design process, it is still important to validate advanced aircraft and component designs experimentally at realistic flight conditions. Such experimental studies would lead to a better understanding of the physics associated with these complex geometries and would contribute to improvements in the CFD tools used in the design process. There also continues to be a need to develop reliable methods for predicting jet/plume viscous interaction effects and to generate the databases necessary to evaluate the performance potential of innovative thrust vectoring and circulation control propulsion integration concepts.

The NTF (Fig. 1) is one of a limited number of wind tunnel facilities that can achieve flight Reynolds numbers and Mach numbers for transport type aircraft for both cruise and high lift operations. The new flow control and propulsion simulation capability at the NTF is focused on the use of semi-span models due to the relative ease of routing high-pressure air to the model and the increased model size compared to a conventional sting mounted fullspan model. The increased model size allows higher fidelity, as well as increased internal volume for housing the flow control mechanisms and instrumentation. For propulsion and/or active flow control testing, the operating envelope of the tunnel is limited to total pressures that

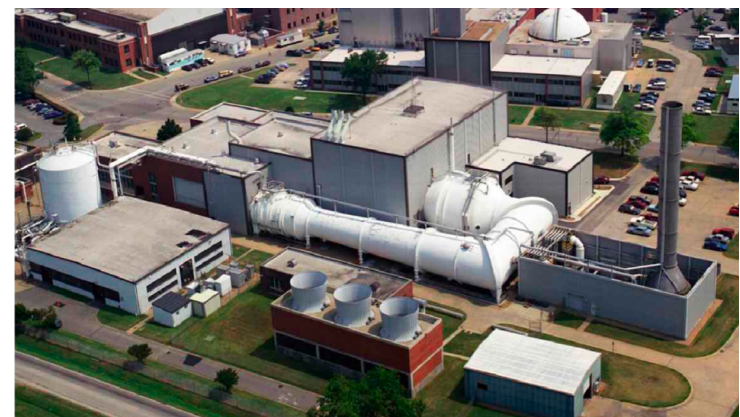

Figure 1. Aerial Photo of NTF. range from 1 to 8 atmospheres and total temperatures of $-50^{\circ} \mathrm{F}$ to $120^{\circ} \mathrm{F}$. These limits are established to minimize the costs associated with customized internal model valve systems and complexities associated with testing at full cryogenic test conditions, such as model frosting. The objectives of the dual nozzle model test were to evaluate the limits of the air station and to obtain estimates of the momentum and pressure tares associated with the bellows interface to the balance. The remainder of this paper will describe the new air supply system and the evaluation of the Dual Aerodynamic Nozzle (DAN) model over a limited portion of the NTF operating envelope.

\section{Experimental Setup}

Two primary objectives of this test series were 1) to provide a simple but safe mechanism that can be used to evaluate the NTF operating envelope of the new dual flow air delivery system, and 2) to evaluate the accuracy of the weight flow measuring system to be used in future propulsion airframe integration and active flow control testing. This section will be divided into descriptions of the wind tunnel, air delivery system, weight-flow metering systems, Sidewall Model Support System (SMSS), and the DAN model. 


\section{Wind Tunnel}

The NTF (Fig. 2) is a fan-driven, closed-circuit, continuous-flow, pressurized wind tunnel capable of operating either in dry air at warm temperatures or in nitrogen gas from warm to cryogenic temperatures. The test section is $8.2 \mathrm{ft}$ by $8.2 \mathrm{ft}$ in cross section and $25 \mathrm{ft}$ in length. The test section floor and ceiling are slotted (six percent open), and the sidewalls are solid. The wind tunnel is capable of an absolute pressure range from one atmosphere to 8.3 atmospheres, a temperature range from $-270^{\circ} \mathrm{F}$ to $130^{\circ} \mathrm{F}$, a Mach number range from 0.1 to 1.2 , and a maximum Reynolds number of $146 \times 10^{6}$ per foot at Mach 1 . Typical tests in this facility use a temperature range from $-250^{\circ} \mathrm{F}$ to $120^{\circ} \mathrm{F}$. For semi-span operations that include propulsion and/or active flow control testing, the operating envelope of the tunnel is limited to total pressures that range from 1 to 8 atmospheres and total temperatures of $-50^{\circ} \mathrm{F}$ to $120^{\circ} \mathrm{F}$.

\section{Dual Flow Air Delivery System}

The new air delivery system is a permanent high-pressure air system that provides a continuous source of clean, dry air. The system was designed to provide a total weight flow of $28 \mathrm{lbm} / \mathrm{sec}$ divided into two flow paths. The dual flow system consists of two independent air supply lines that are tied to the SMSS used for semi-span testing. A schematic of a single leg of this system is shown in Fig. 3. Each leg is equipped with a coarse and fine control valve and has a corresponding vortex flow metering system. A multiple critical venturi (MVC) system was installed in the later stages of the test to supplement the evaluation of the vortex flow metering system. The total temperature of the air stream can be set from $0^{\circ} \mathrm{F}$ to $120^{\circ} \mathrm{F}$ by using a steam heating system. The low temperature settings are dependent on Joule Thompson effects and thermal conduction associated with the piping located in the low temperature environment of the wind tunnel plenum.

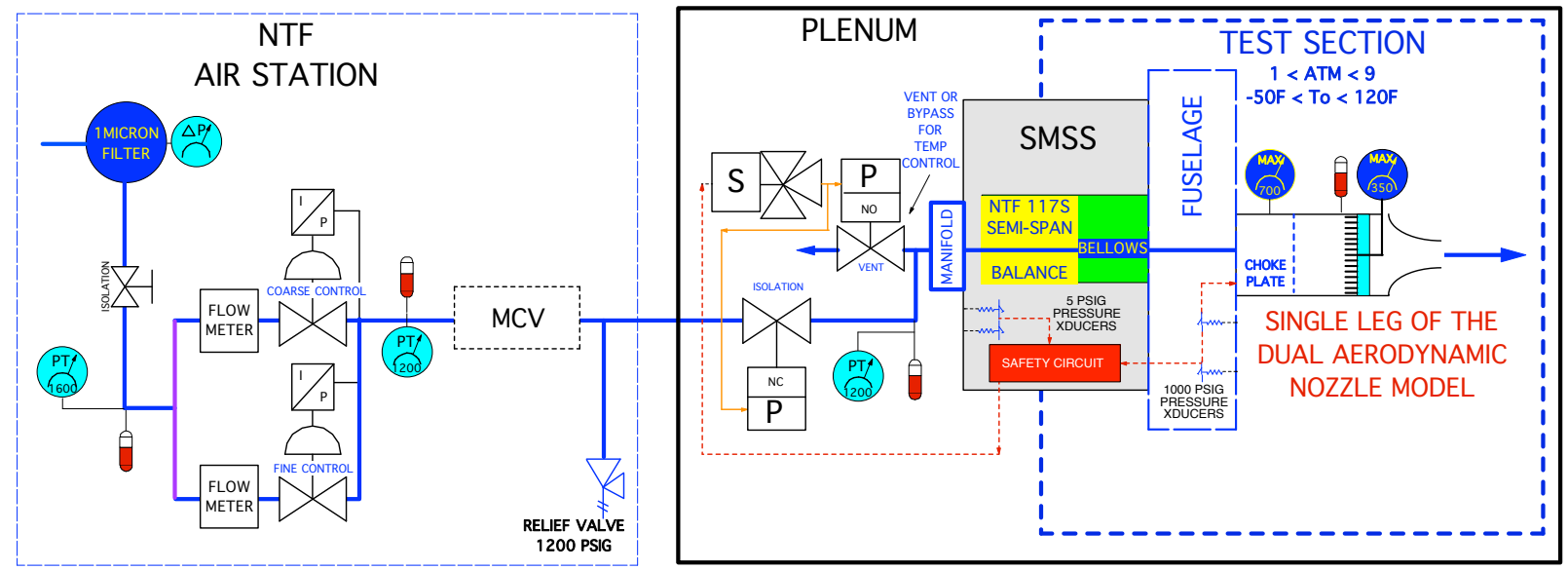

Figure 3. Sketch of a single leg of the NTF air station.

The high-pressure air station also incorporates a model protection system that will limit the maximum pressure delivered to the model. The maximum pressure limit can be adjusted from 300 psi to 1200 psi. For the DAN model 
a 1200 psig limit was established to protect the model in the unlikely event that the model choke plate should experience a blockage, like liquid freezing in the choke plate holes. If the pressure matches or exceeds the limit, then the supply line into the SMSS would be isolated and vented in less than 0.5 seconds.

\section{Mass Flow Metering System}

The weight-flow metering system was designed around four vortex mass flow meters that were installed upstream of the fine and coarse control valves in each of the two flow legs of the air station. These flow meters were calibrated by the Colorado Engineering Experiment Station, Inc. (CEESI) in April 2010. A first order linear fit was applied to the calibrated flow meter sensitivity (Kfactor) resulting in a reduction in the uncertainty from $\pm 2 \%$ to less than $\pm 0.35 \%$ of reading. A typical error profile for one of the vortex flow meters is shown in Fig. 4.

To ensure that the vortex flow meters were optimized for NTF operations, they were installed with straight sections of pipe both upstream and downstream of the vortex flow meter. The 50:1 inlet pipe diameters and a 20:1 exit pipe diameters exceeded the standards for typical vortex flow meter systems. This configuration was designed to minimize vibrations and ensure uniformity thru the system. Vibrations did occur at the several of the flow meter locations and were determined to adversely affect the uncertainty of the metering system. This issue will be discussed later in the paper.

To validate the vortex flow meter system, a MCV calibration standard, shown in Fig. 3, was placed in series with the low flow leg of the air station. This flow measurement system can accommodate up to $44 \mathrm{lbm} / \mathrm{sec}$ of air at a maximum inlet pressure of 1500 psi. As shown in Fig. 5, the system inlet flow is distributed uniformly into a common plenum by a radial inlet diffuser and a large perforated plate. The flow then passes thru a combination of calibrated venturis before exiting the system. The venturis vary in size in binary increments of throat area so that each successively larger venturi will pass twice the flow of the preceding one. The unused venturis are typically capped to force all of the flow thru the remaining venturis. The combined uncertainty of the MCV system is reported to be less than 0.1 percent of reading.

\section{Sidewall Model Support System (SMSS)}

The primary function of the SMSS is to support the model, balance hardware, flow control piping, and electronics in the test section wall. A 5-component balance is mounted inside the SMSS as shown in Fig. 6 and its maximum calibrated load limits are highlighted in Table 1. The SMSS is a heated enclosure that maintains a stable temperature for the balance and the pitch mechanism. The high pressure air enters the SMSS via two independent manifolds that are connected to a rotary union that allows the mechanism to pitch. The air then passes through the center of the balance through concentric air lines where it transitions across the balance via

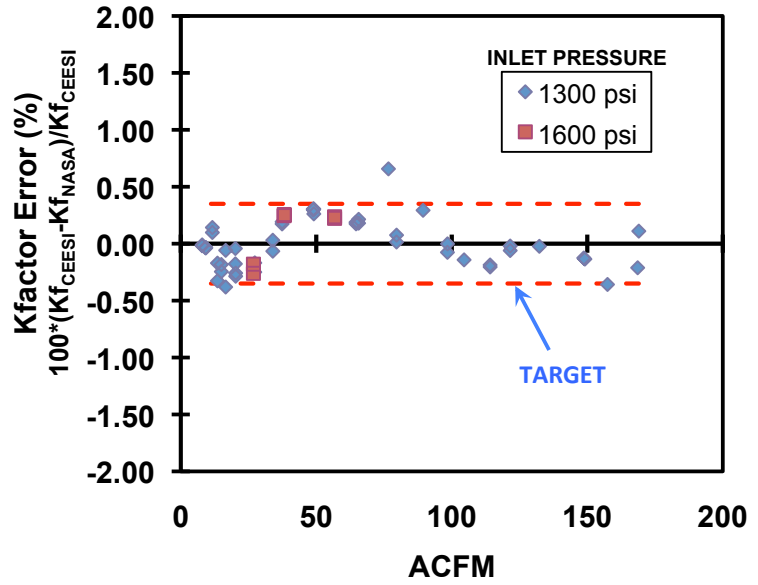

Figure 4. Error profile for high flow vortex flow meter based on CEESI calibration.

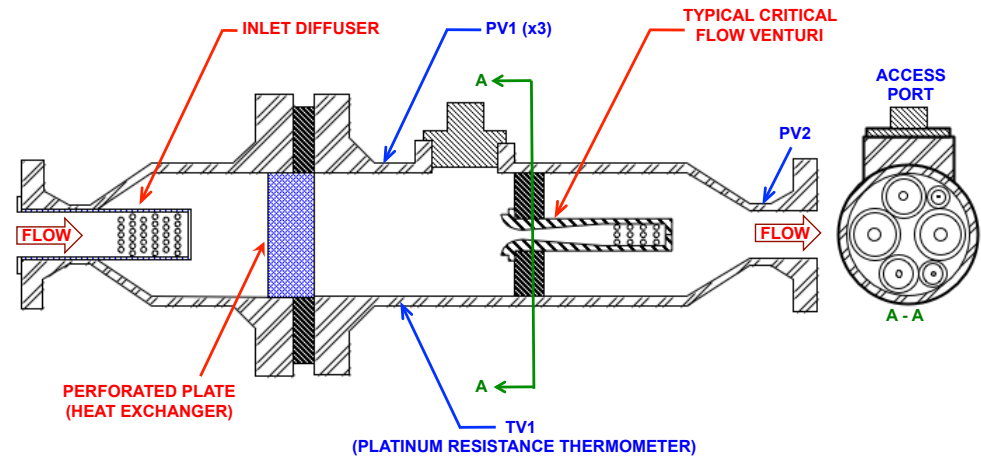

Figure 5. Sketch of MCV, highlighting the orientation of the 6 critical flow venturis. 
independent high-flow and low-flow bellows. Each of these bellows are designed to minimize the balance tares and momentum transfer caused by the high pressure air crossing the metric/non-metric boundary in the air delivery system.

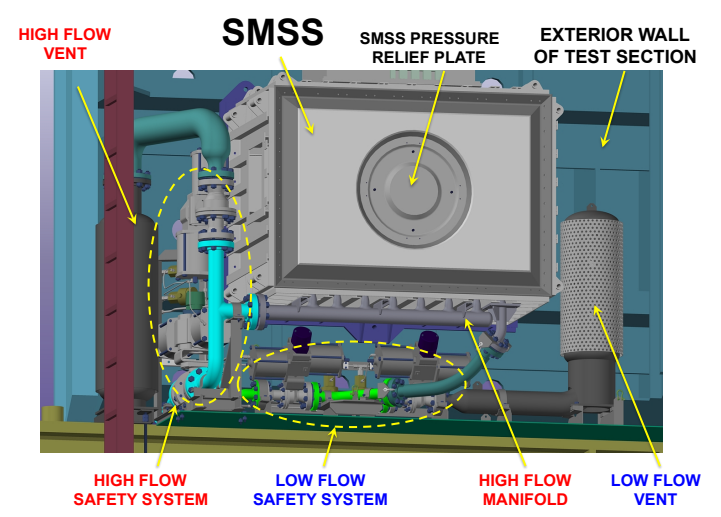

(a) SMSS (interior plenum view)

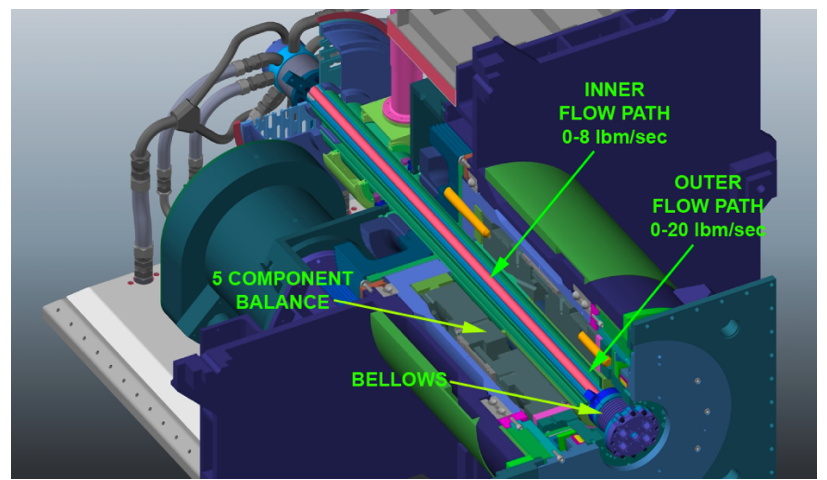

(b) Cutaway sketch of SMSS

\section{Figure 6. Sketch of the NTF SMSS installation, highlighting the safety systems and interior view of the} balance and concentric flow paths.

To characterize the pressure tare, the model was loaded with known weights in the normal force, axial force, and pitching moment balance components with the air system off to determine the balance and model installation effects. A limited series of calibration runs were also performed with the system pressurized to determine the pressurization effects of the bellows. These pressure tares are subtracted from the balance data to obtain aerodynamic loads.

\section{Dual Aerodynamic Nozzle (DAN) System}

The primary design requirement for the DAN model, shown in Fig. 7, was to vector a high-flow source of air (20 $\mathrm{lbm} / \mathrm{sec}$ at $1200 \mathrm{psig})$ and an independent low-flow source of air (8 lbm/sec at $1200 \mathrm{psig})$ along the streamlines of the tunnel to avoid jet impingement on the instrumented tunnel walls. While the model design criteria included operation at the maximum pressure limits of the facility, it was limited to operating temperatures in the range of $-50^{\circ} \mathrm{F}$ to $120^{\circ} \mathrm{F}$.

The DAN design was based on existing Stratford Choke Nozzles ${ }^{4}$ obtained from NASA Langley's Jet Exit Facility. Twelve interchangeable Stratford nozzles that range from 0.999 $\mathrm{in}^{2}$ to $11.352 \mathrm{in}^{2}$ are available for use with this model. These nozzles ${ }^{5}$ were used at the Jet Exit Facility and the 16-Foot Transonic Tunnel to obtain balance tares resulting from airflow momentum and pressure. 6

The two Stratford nozzles used as part of this investigation are consistent with typical nozzle areas anticipated in future propulsion simulation and active flow control geometries in the NTF. The minimum throat area, $A_{t}$, occurs at the Stratford jet exit where the flow will

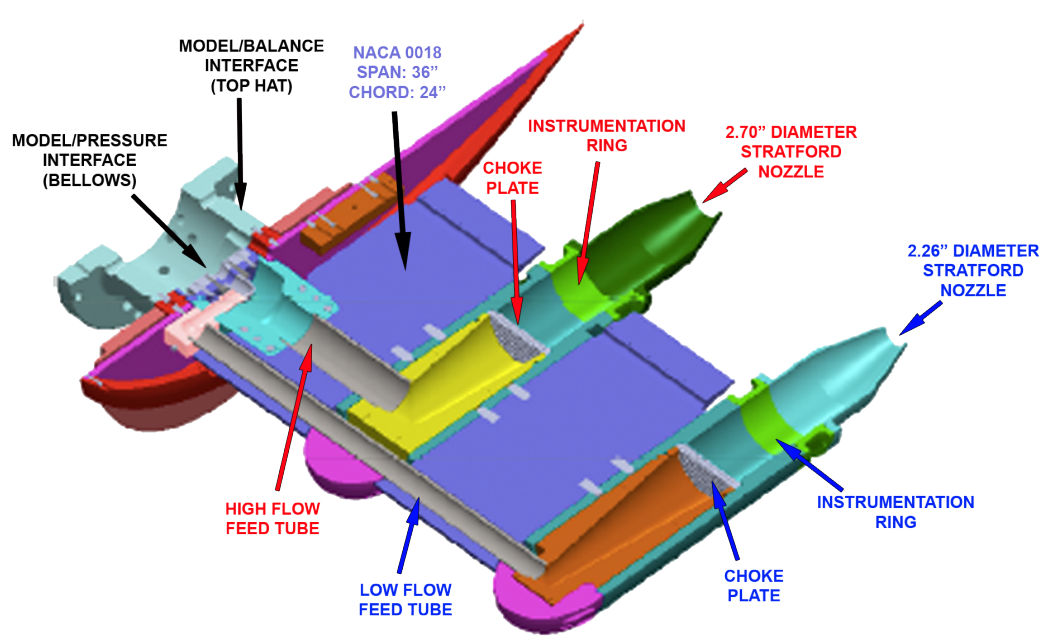

Figure 7. Cutaway sketch of the DAN Model. become sonic. The high-flow leg utilized 
the Stratford nozzle having a throat area of $5.711 \mathrm{in}^{2}$ (diameter of 2.696 inches) and the low-flow leg utilized the Stratford nozzle having a throat area of $4.005 \mathrm{in}^{2}$ (diameter of 2.26 inches).

\section{Choke Plate Design}

The nozzle flow passes through an interchangeable choke plate and instrumentation section, then into the Stratford nozzle shown in Fig. 8. This arrangement promotes uniform flow and provides flow metering to the nozzle. The choke plate is a perforated disk with the upstream end of each hole countersunk to improve efficiency. The choke plate design was based on an empirical approach described in Reference 7. The design requirements for the choke plate were based on a maximum inlet pressure of $1200 \mathrm{psig}$ and a maximum flow rate of $20 \mathrm{lbm} / \mathrm{sec}$ for the high-flow

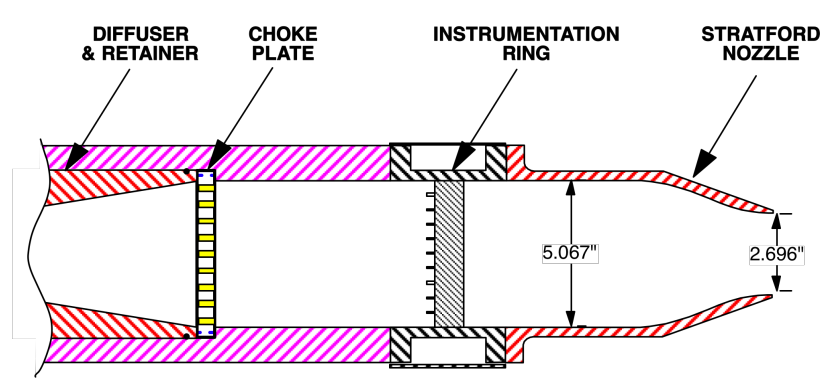

Figure 8. Sketch of Stratford nozzle assembly. nozzle and $8 \mathrm{lbm} / \mathrm{sec}$ for the low-flow nozzle. The upstream choke plate pressure was monitored by the air station safety circuit and was designed to automatically isolate and vent the air station in the event of over pressurization within the model. An estimate of the upstream pressure was determined from pressure losses described in Equation 1. Two choke plate designs were established for each nozzle used during this test. The test section static pressure and the NPR influence the upstream choke pressure as shown in Fig. 9a and $9 \mathrm{~b}$ for wind-off tunnel conditions. The 36\% open area choke plate design was intended to minimize the risk of plugging the choke holes in the event icing or some other type of blockage while attempting to minimize the pressure losses and straighten the flow into the nozzle. The $15 \%$ open area choke plate was designed to remain choked throughout the test envelope thereby enhancing flow uniformity into the nozzle. The upper limits of either choke plate design are established by the maximum flow through the nozzle. Static pressures above 5 atmospheres are limited to subsonic flow for both designs. The 36\% open area choke plate was chosen for this test series based on a more conservative safety analysis.

$$
P_{T(C H O K E)}=\frac{\rho_{\text {CHOKE }} U_{C H O K E}^{2}\left[0.5(1-\bar{f})^{0.75}+\tau(1-\bar{f})^{1.375}+(1-\bar{f})^{2}+\lambda \frac{1}{d_{o}}\right]}{2 k_{M}(\bar{f})^{2}}-P_{T(J E T)}(N P R)^{2}
$$

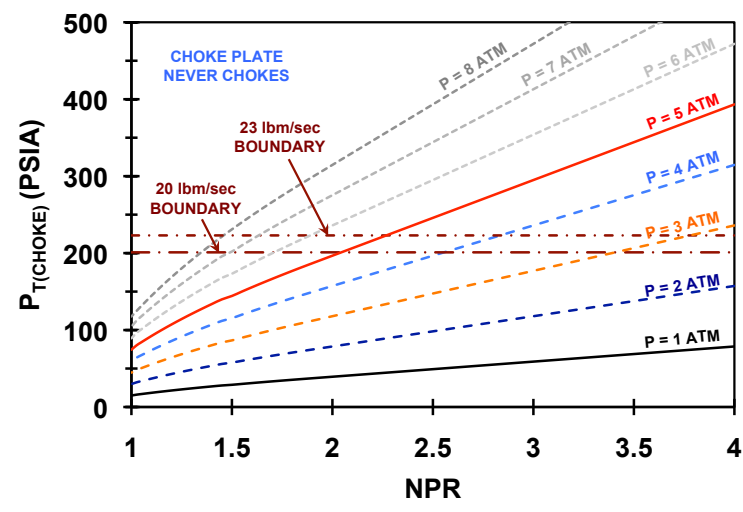

(a) $\bar{f}=0.36, d_{0}=0.1936$ in., 246 orifices

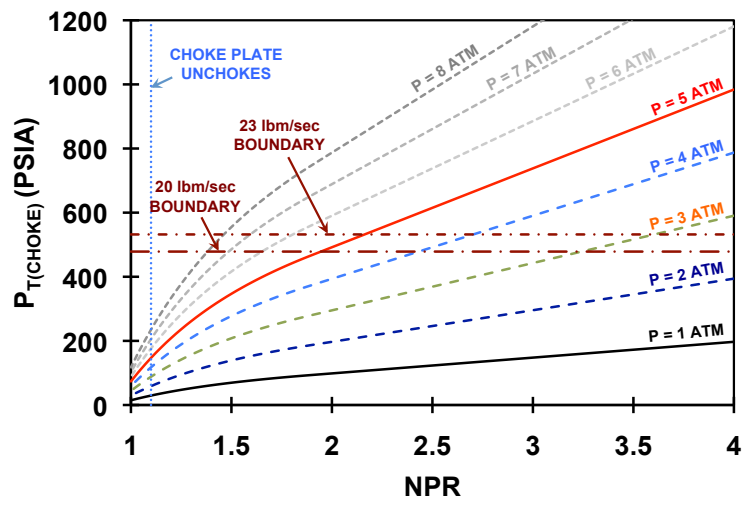

(b) $\bar{f}=0.15, d_{0}=0.125$ in., 246 orifices

Figure 9. Estimates of the upstream pressure for the high flow leg choke plate, $T_{T(J E T)}=0^{\circ} F, d=2.696$ in. 


\section{Instrumentation Section}

The instrumentation ring of each leg of the DAN model housed a 9-port total pressure rake and two total temperature probes. The internal pressure rake was aligned with the horizontal axis and the total temperature probes were 67.5 degrees off axis and 180 degrees from each other shown in figure 10. The probe tips of the total pressure rake were located 50 hole-diameters downstream of the choke plate orifices to ensure that the flow is fully developed at the measurement location. Initial testing utilized only the centerline port of the rake due to limitations placed on the use of heated enclosures which housed 64-port electronically scanned pressure system

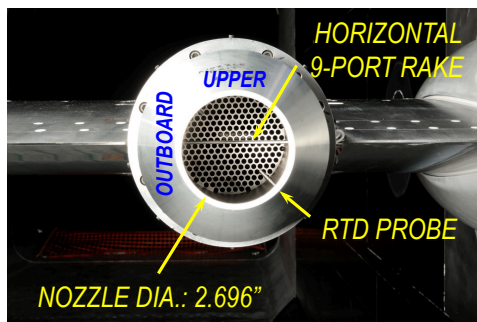

Figure 10. Photo of nozzle exit. normally used for this type of application. A single 100-psid pressure transducer having a resolution of \pm 0.1 psi was used for this measurement. Since the transducer was located in the fuselage without a heater it was calibrated over a pressure range of 0 to $100 \mathrm{psig}$ and temperature range of $-50^{\circ} \mathrm{F}$ to $120^{\circ} \mathrm{F}$.

In addition to the internal rake, the jet total-pressure distribution at the nozzle throat was determined for each configuration tested with a 64-port rake located 0.05 inches downstream of the jet exit or nozzle throat. The probes on the rake were spaced 0.01 inches apart. This rake was mounted on a 2 -axis traversing system with a 0.00010 inch step resolution. Since the traversing system was temporarily mounted in the test section without fairings, the jet profile measurements used to evaluate nozzle performance were limited to wind tunnel fan-off conditions at one atmosphere. This allowed the use of a 30-psid electronically scanned pressure system having a resolution of \pm 0.015 psi to be used for the rake surveys. All nine ports from the rake were manifolded together and attached to the single 100-psid transducer after the external rake survey was completed.

\section{Stratford Choke Nozzles}

Gas-flow is typically measured by metering systems that, in general, cannot be calibrated by direct weighing of the flow per unit time as in liquid flow systems. Therefore secondary standard nozzles are employed to calibrate weight-flow rate measurements. These data can be used to obtain balance tares resulting from airflow momentum and pressure losses. The secondary standard nozzles used in the NTF are choke (sonic) nozzles of the type described in reference 6 . The minimum diameter that forms the throat of the Stratford nozzle occurs at the exit plane of the nozzle. The Stratford choke nozzle having a throat area of $\mathrm{A}_{t}=5.711 \mathrm{in}^{2}$ was used in this NTF test series and was calibrated against several primary standard nozzles at the CEESI complex in 1968. The CEESI primary standard nozzles have known discharge coefficients that have been verified by the National Bureau of Standards.

To characterize the flow metering system associated with the air delivery system it is necessary to understand the performance of the Stratford nozzles without any external flow. This establishes an ideal weight flow and thrust that can be used to understand the performance associated with the SMSS and bellows interface to the balance. Once these tares are determined, they can be applied to any model configuration.

The DAN model is being established as a reference model for blowing configurations at NTF. The model is limited to a dynamic pressure of 1000 $\mathrm{lb} / \mathrm{ft}^{2}$ and therefore the model-operating envelope only covers a limited portion of the facility-operating envelope shown in

\section{Results}

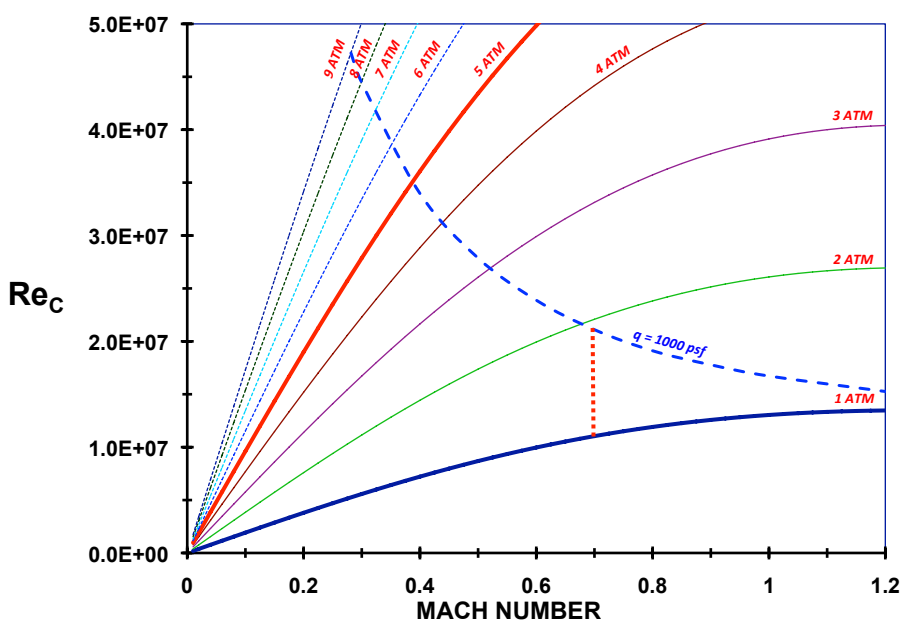

Figure 11. The DAN model operating envelope, reference chord $=24$ in., $T_{T}=-50^{\circ} \mathrm{F}$ 
Fig. 11. Three wind tunnel Mach numbers were chosen as part of this evaluation, Mach $=0.2,0.4$, and 0.7. The corresponding total pressures are limited to 5 atmospheres, 3.2 atmospheres, and 1.9 atmospheres.

\section{Flow Meter Performance}

The performance of the two Stratford nozzles used as part of this investigation is described in references 4 and 5 as a function of the nozzle discharge coefficient and nozzle pressure ratio, NPR. The nozzle discharge coefficient is defined as:

$$
C_{D I S}=\frac{w_{P}}{w_{I}}
$$

The losses characterized by the discharge coefficient can be attributed to flow non-uniformity associated with the upstream choke plate performance and internal viscous effects. These parameters are sensitive to Reynolds number as described by Stratford in Reference 4. The measured weight flow, $\mathrm{w}_{\mathrm{P}}$ is measured using a vortex flow meter and MCV that is part of the NTF air station. The ideal weight flow was determined using Eq. (3) and is a function of the nozzle pressure ratio, NPR.

For NPR $<(\mathrm{NPR})_{\text {CRITICAL, }}$

$$
w_{I}=P_{T(J E T)} A_{t}\left(\frac{1}{N P R}\right)^{\frac{1}{\gamma}} \sqrt{\frac{2 g \gamma}{(\gamma-1) R T_{T(J E T)}}\left(1-\left(\frac{1}{N P R}\right)^{\frac{\gamma-1}{\gamma}}\right)}
$$

and for NPR $>(\mathrm{NPR})_{\mathrm{CRITICAL}}$,

$$
w_{I}=P_{T(J E T)} A_{t} \sqrt{\frac{g \gamma}{R T_{T(J E T)}}\left(\frac{2}{\gamma+1}\right)^{\frac{\gamma+1}{\gamma-1}}}
$$

where NPR is:

$$
N P R=\frac{P_{T(J E T)}}{P}
$$

and $(\mathrm{NPR})_{\mathrm{CRITICAL}}=1.8926$.

The ideal weight flow for several different wind tunnel conditions is shown in Fig. 12. These data were used to predict the boundaries required to maintain sonic conditions at the nozzle throat for different wind tunnel conditions.
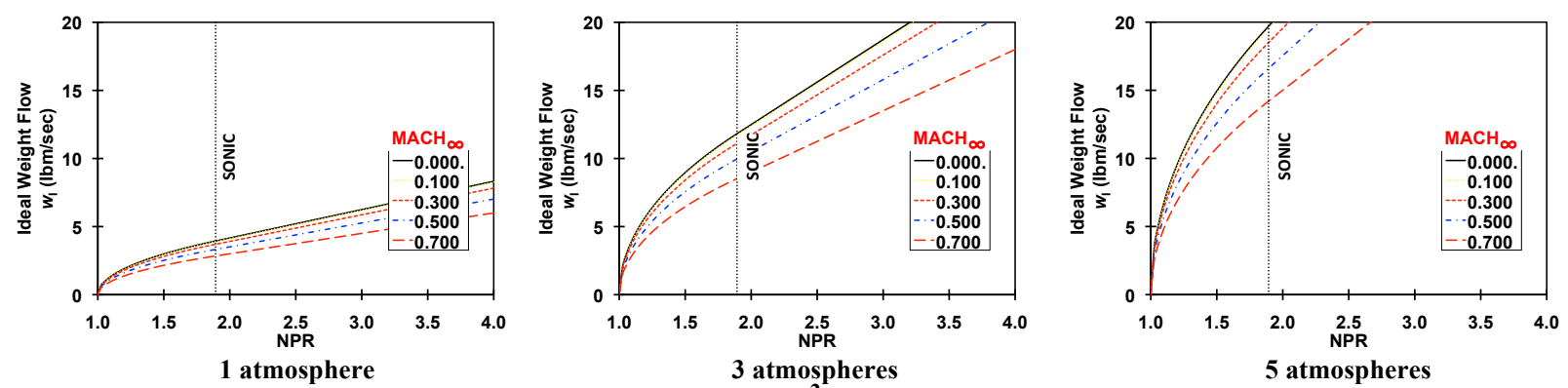

Figure 12. Ideal Weight Flow, Nozzle exit area $=5.711 \mathrm{in}^{2}, \mathrm{~T}_{\mathrm{T}}=-50^{\circ} \mathrm{F}, \mathrm{T}_{\mathrm{T}(\mathrm{JET})}=0^{\circ} \mathrm{F}$.

These results indicate that sonic conditions can be achieved for tunnel pressures up to five atmospheres and are limited only by the available flow rate of $20 \mathrm{lbm} / \mathrm{sec}$. The choked flow discharge coefficient for the $5.711 \mathrm{in}^{2}$ Stratford nozzle obtained in the 16' Transonic Tunnel (TT) using a MCV is described in Ref. 5 and is shown in Fig. 13 along with data from the current test. After comparing current results using the vortex flow meter system to the 16'TT Stratford nozzle data, it was evident that the nozzle performance needed to be re-examined. This decision was reinforced by the poor performance of the choke plate that was designed to promote flow uniformity. The measured change in pressure across the choke plate was not consistent with a choked condition. This indicated that the internal flow path was compromised and that the measured total pressure was biased. 
The measurement uncertainty of the nozzle total pressure required to compute the ideal weight flow is affected by the 3-D non-uniformity of the flow at the nozzle exit shown in Fig. 14. The poor measurement resolution near the nozzle boundaries is not acceptable to perform an independent integration that could be used for characterizing the total weight flow; however, it is adequate for assessing the core of the jet and obtaining corrections to the total pressure measurements. These data acquired at one atmosphere highlight the nozzle nonuniformity that can be attributed to the losses associated with the final turning of the flow as it passes into the inlet of the nozzle plenum.

To assess the total pressure correction of the rake located in the instrumentation ring, the throat pressure profile was averaged at the rake station, $(\mathrm{Y}=0)$. The integration of the data was bounded to include only the

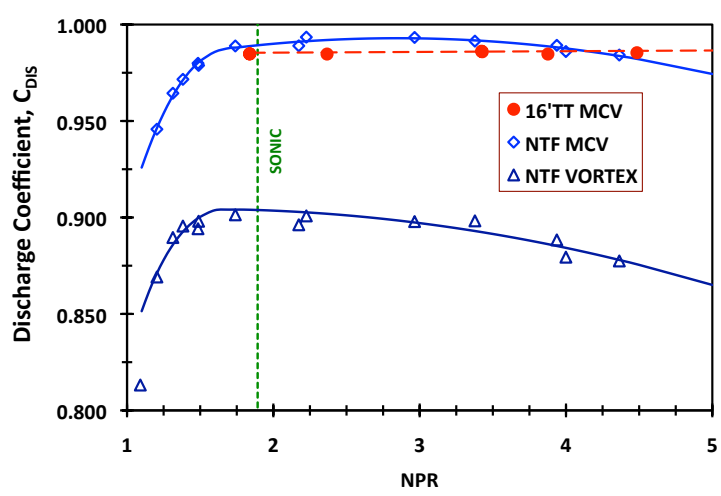

Figure 13. Comparison of nozzle discharge coefficients for the NTF flow meters system, nozzle exit area $=5.711 \mathrm{in}^{2}, \mathrm{~T}_{\mathrm{T}}=51^{\circ} \mathrm{F}, \mathrm{T}_{\mathrm{T}(\mathrm{JET})}=$ $-8^{\circ} \mathbf{F}$.

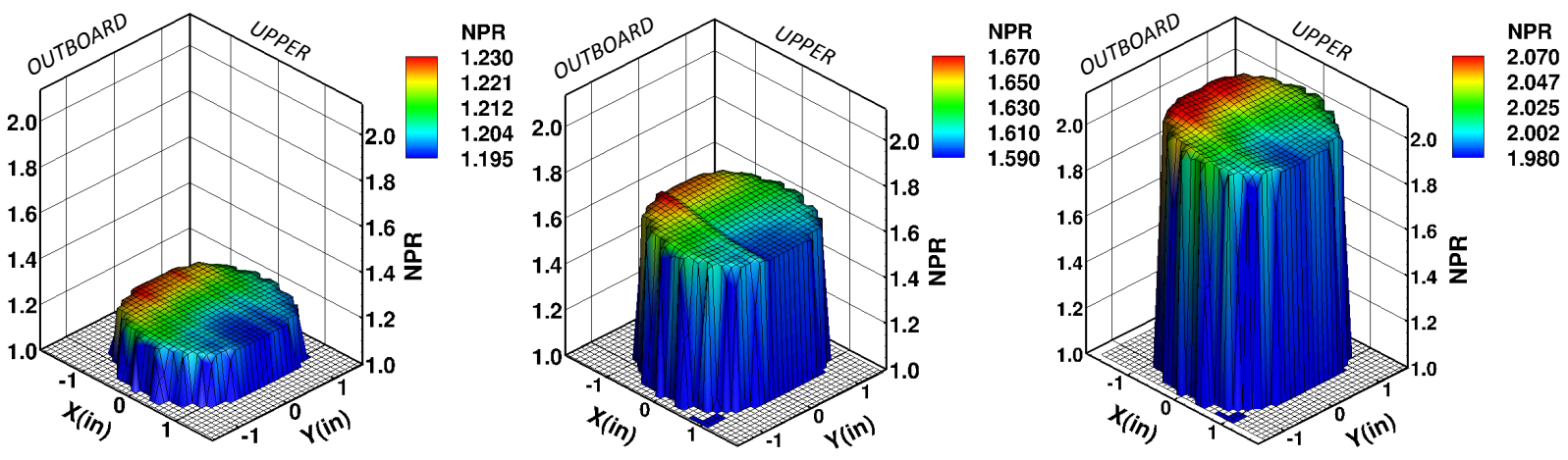

Figure 14. Pressure profiles at the throat (exit) of the inboard nozzle $\left(A_{t}=5.711 \mathrm{in}^{2}\right)$, NPR $=1.2,1.6,2.0$.

core of the jet. The average pressure was consistently higher than the single measurement made on the centerline using the internal rake. The nozzle total pressure corrections increase linearly until the nozzle chokes, as highlighted in table 2. Applying these corrections did not significantly improve the vortex flow discharge coefficients shown in Fig. 13. Further investigation indentified that flow induced vibrations in the supply piping adjacent to the vortex flow meters exceeded $20 \mathrm{~g}$ 's. Since this exceeded the manufacturer's specifications by a factor of 20 , these vibrations were determined to be a major source of the vortex flow meter inaccuracies.

To verify the Stratford nozzle weight flow measurements and quantify the vortex flow meter inaccuracies, a secondary flow standard (MCV), shown in Fig. 3, was placed in the flow path. The MCV discharge coefficients shown in Fig. 13 are consistent with the 16'TT data at low supersonic conditions and indicate that the instrumentation ring total pressure rake

Table 2. Nozzle total pressure corrections, 1 atm.

\begin{tabular}{|c|c|c|c}
\hline NPR & AVG NPR $(Y=0)$ & Correction (\%) & STD/AVG (\%) \\
\hline 1.2015 & 1.2073 & 0.4784 & 0.3869 \\
\hline 1.4130 & 1.4259 & 0.9146 & 0.3179 \\
\hline 1.6060 & 1.6215 & 0.9663 & 0.4997 \\
\hline 1.7982 & 1.8265 & 1.5721 & 0.5316 \\
\hline 2.0220 & 2.0257 & 0.1813 & 0.3501 \\
\hline
\end{tabular}
corrections are valid up to NPR $=2$.

These independent tests also confirmed that there was a problem with the vortex flow meters resulting in a biased weight flow that is approximately $10 \%$ lower than the weight flow obtained using the MCV. The uncorrected vortex meter weight flow data was used to characterize the boundaries of the dual flow air station. As a result, the actual measured flow rate thru the DAN model exceeded the predicted design flow rate for both the high flow and low flow geometries, i.e., the high flow leg peaked at $23 \mathrm{lbm} / \mathrm{sec}$ (corrected to $25 \mathrm{lbm} / \mathrm{sec}$ ) and the low flow leg peaked at $9 \mathrm{lbm} / \mathrm{sec}$ (corrected to $10 \mathrm{lbm} / \mathrm{sec}$ ). Since the completion of this test, the air station has been re-configured with dedicated MCV's. 
Characterizing the discharge coefficients for different free stream Mach numbers required that a correction be applied to the discharge coefficient due to vortex meter errors and total pressure errors related to nozzle flow non-uniformity. A single constant was added to the discharge coefficients to bring the magnitudes into alignment with the wind-off baseline data described above. The analysis is therefore limited to relative changes in nozzle performance. This approach does not account for changes in local pressure fields associated with wind tunnel wall interference or model blockage.

The impact of free stream Mach number on nozzle discharge coefficient is shown in Fig. 15. The inboard nozzle discharge coefficient was reduced by $3.5 \%$ as the free stream Mach number increased from 0.2 to 0.7 and $4.7 \%$ for the outboard nozzle. The variation in discharge coefficients for elevated free stream Mach numbers can be related to the difference in the jet exit static pressure and the free stream static pressure. These pressure differences can be linked to the separation region in the outer flow just upstream of the jet exit.

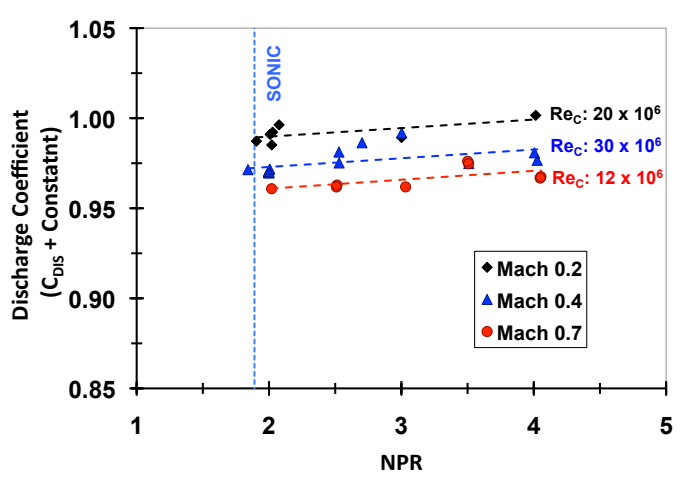

a) Inboard nozzle, $A_{t}=5.711$ in $^{2}$

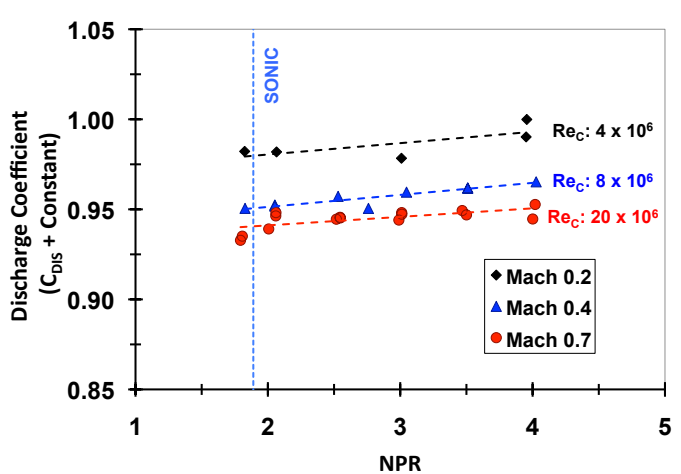

b) Outboard nozzle, $A_{t}=4.000$ in $^{2}$

Figure 15. Comparison of nozzle discharge coefficients for different Mach numbers, $\mathrm{T}_{\mathrm{T}}=-\mathbf{5 0} 0^{\circ} \mathrm{F}$.

\section{NTF-117S Balance and Bellows Performance}

While the primary objective of the test series was to evaluate the flow metering system, it was also an opportunity to obtain preliminary data on the new NTF $117 \mathrm{~S}$ balance and to establish test procedures associated with the bellows. The bellows was designed to limit the impact of the air delivery system onto the balance. Two primary parameters that influence the bellows interface are pressure and temperature. Although these parameters are expected to be small due to the stiffness of the balance, procedures were developed to account for both pressure and temperature. Since the bellows is located at the interface of the SMSS and the model, there is a temperature gradient that can be as large a $150^{\circ} \mathrm{F}$. The wind tunnel total temperature, the SMSS temperature, and the jet total temperature influence this temperature gradient. Initial testing revealed the need to pre-condition the bellows temperature. These temperature effects were addressed by flowing air thru the bellows at the desired temperature prior to obtaining a wind-off zero on the balance.

The influence of the pressure applied thru the bellows onto the balance changes at every NPR. This requires an extensive load calibration over the entire pressure range of the system. Pressure tares were obtained during check loading of the model in the test section; however, this data set was limited due to the weight restrictions on the test section loading apparatus. A post balance calibration must be performed before final force and moment results can be obtained.

Temperature and pressure effects on the bellows can also affect the measured thrust efficiency of the nozzle. The thrust efficiency of a nozzle is determined by dividing the measured thrust, $F_{R}$, by the ideal isentropic thrust, $F_{I}$ to obtain the resultant thrust ratio, $\mathrm{F}_{\mathrm{R}} / \mathrm{F}_{\mathrm{I}}$. The measured resultant thrust is given by:

$$
F_{R}=\sqrt{F_{A X I A L}^{2}+F_{N O R M A L}^{2}}
$$

In general the thrust ratio is a measure of nozzle efficiency and is dependant on the plume shape of the nozzle as shown in Fig. 16. For a critical nozzle that is optimized at a Mach number of one, the static pressure at the jet exit is equal to the free stream static pressure. 

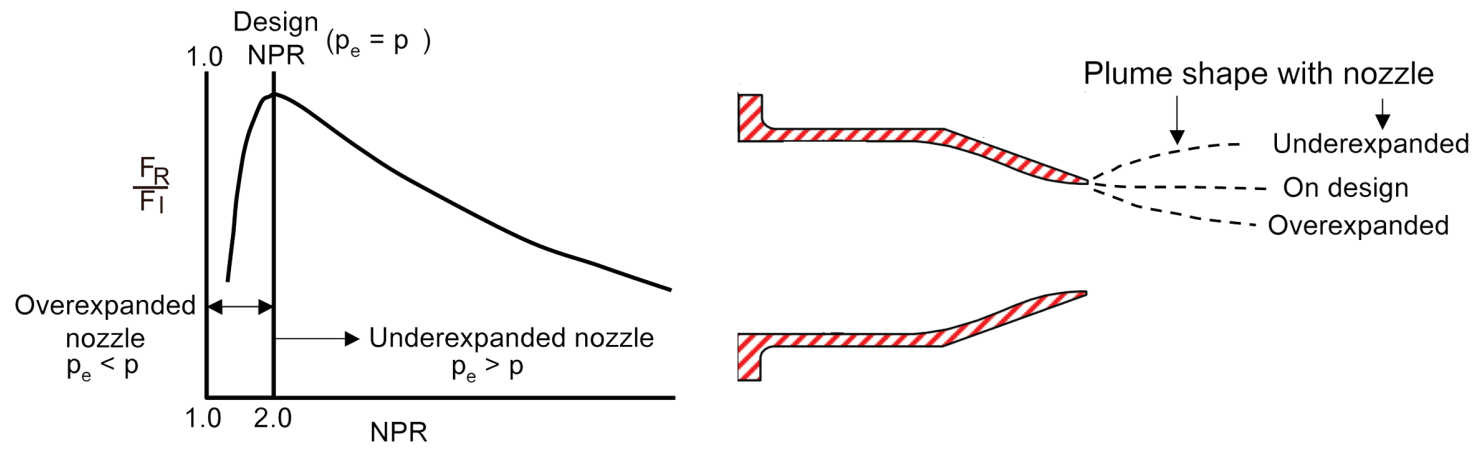

Figure 16. Thrust ratio for an over-expanded and under-expanded nozzle.

For the DAN model, the measured resultant thrust can be obtained from the balance data acquired when the model was set to an angle-of-attack of zero, resulting in all of the thrust forces acting in the axial direction. The ideal isentropic thrust used in the thrust ratio evaluation is calculated assuming a one-dimensional isentropic expansion from measured stagnation conditions and the nozzle pressure ratio,

$$
F_{I}=w_{P} \sqrt{\frac{2 \gamma R T_{T(J E T)}}{g(\gamma-1)}\left[1-(N P R)^{\frac{1-\gamma}{\gamma}}\right]}
$$

Figs. 17 and 18 show the ideal thrust for the DAN nozzles to be within the range of the axial component of the balance for NPRs up to four. The measured maximum NPR was limited by the flow rate and occurred at an NPR of 11.2 at a weight flow of $23 \mathrm{lbm} / \mathrm{sec}$ for the inboard nozzle and an NPR of 6.2 at weight flow of $9 \mathrm{lbm} / \mathrm{sec}$ for the outboard nozzle. Both nozzles were also run simultaneously and achieved a combined weight flow of $32 \mathrm{lbm} / \mathrm{sec}$.
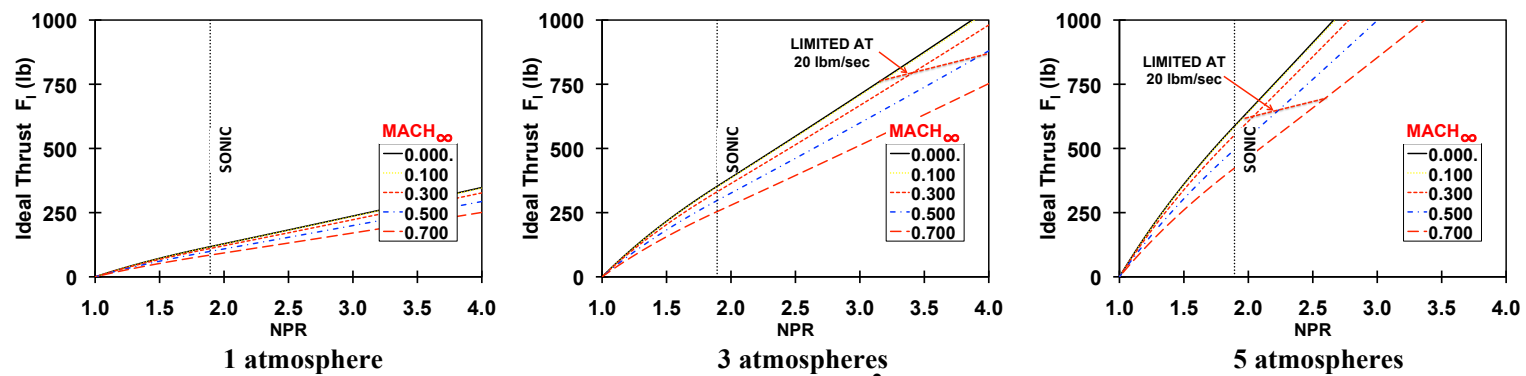

Figure 17. Ideal Thrust, Inboard Nozzle exit area $=5.711 \mathrm{in}^{2}, \mathrm{~T}_{\mathrm{T}}=-50^{\circ} \mathrm{F}, \mathrm{T}_{\mathrm{T}(\mathrm{JET})}=0^{\circ} \mathrm{F}$.
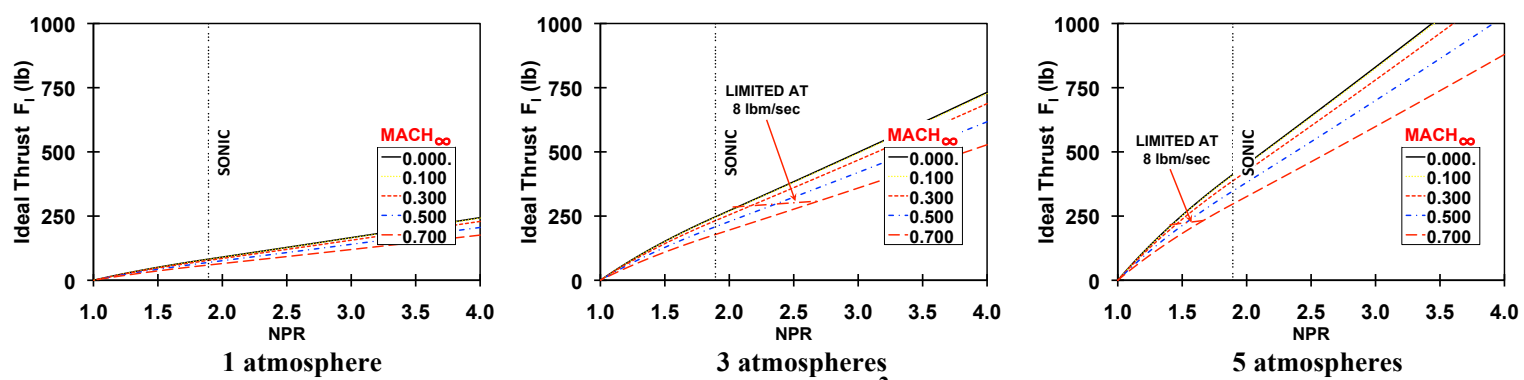

Figure 18. Ideal Thrust, Outboard Nozzle exit area $=4.00 \mathrm{in}^{2}, \mathrm{~T}_{\mathrm{T}}=-50^{\circ} \mathrm{F}, \mathrm{T}_{\mathrm{T}(\mathrm{JET})}=0^{\circ} \mathrm{F}$. 
The thrust ratio for the $5.711 \mathrm{in}^{2}$ Stratford nozzle was obtained in the 16'TT tunnel as part of their air delivery and balance calibration, shown in Fig. 19. These data will be the reference used to compare with the NTF data set. Preliminary NTF results indicate that the thrust efficiency of the DAN model is lower than expected, largely due to the bellows pressure and temperature effects. An effort is currently underway to compensate for these effects that will include an extended post-test balance calibration to obtain the pressure tare coefficients.

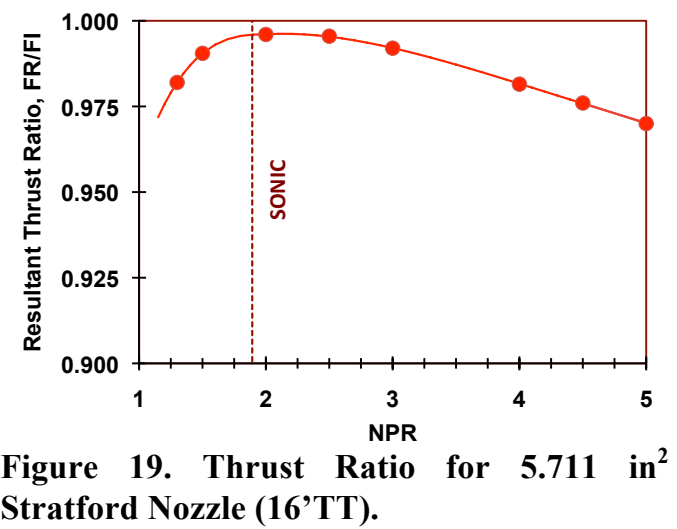

\section{Concluding Remarks}

The DAN model was used to demonstrate and evaluate performance characteristics of a new dual air delivery system for the NTF Facility. This test series identified weight flow and jet temperature boundaries for the air station over a range of tunnel total pressures that varied from 1 atmosphere to 5 atmospheres and tunnel total temperature that varied from $-50^{\circ} \mathrm{F}$ to $120^{\circ} \mathrm{F}$. The weight flow boundaries for the air station was measured to be 0.1 to 23 $\mathrm{lbm} / \mathrm{sec}$ for the high flow leg and 0.1 to $9 \mathrm{lbm} / \mathrm{sec}$ for the low flow leg. The jet temperatures were controlled with a steam heater system and could be set to within $\pm 2^{\circ} \mathrm{F}$ over a range of $-8^{\circ} \mathrm{F}$ to $120^{\circ} \mathrm{F}$.

The results of this effort have highlighted the critical measurement requirements associated with blowing systems used for high Reynolds number testing at the NTF. The Stratford nozzles used in the DAN model can be used as a flow meter standard and thrust calibration standard. However, measured non-uniformities at the throat of the Stratford nozzle can bias the total pressure measurements used to calculate NPR, ideal weight flow, and ideal thrust. These non-uniformities can be attributed to the choke plate design and the flow path through the model.

Comparison of nozzle discharge coefficients of the Stratford nozzle, MCV flow meter, and NTF vortex flow meter revealed problems with the NTF vortex meter system that were traced to excessive vibration in the supply piping. This was found to have a considerable effect on the nozzle discharge coefficient so the vortex flow meter system was replaced with a dual flow MCV system at the end of this test.

Since this was the first application of the NTF dual flow air station, operational procedures were developed and refined throughout this test. These procedures included system leak checks, balance calibrations to include pressure tares, and bellows temperature management. The procedures and data set from this test will provide a guide for future propulsion and active flow control testing in the NTF.

\section{References}

\footnotetext{
${ }^{1}$ Milholen, W. E., Jones, G. S., Cagle, C.M., "NASA High-Reynolds Number Circulation Control Research Overview of CFD and Planned Experiments (Invited)," AIAA 2010-344, January 2010

2 Jones, G.S., Joslin, R.D., "Proceedings of the 2004 NASA/ONR Circulation Control Workshop", NASA/CP-2005213509, June 2005

${ }^{3}$ Zeune, C. H., "Enabling Speed Agility for the Air Force," AIAA 2010-349, January 2010

${ }^{4}$ Stratford, B. S., "The Calculation of the Discharge Coefficient of Profiled Choked Nozzles and the Optimum Profile for Absolute Air Flow Measurement," J.R. Aeronaut. Soc, vol. 68, no. 640, pp. 237-245, April 1964

${ }^{5}$ Berrier, B.L., Leavitt, L.D., and Bangert, L.S., "Operating Characteristics of the Multiple Critical Venturi System and Secondary Calibration Nozzles Used for Weight-Flow Measurements in the Langley 16-Foot Transonic Tunnel," NASA TM 86405, 1985

${ }^{6}$ Capone, F. J., "Static Performance of Five Twin-Engine Non-axisymmetric Nozzles with Vectoring and Reversing Capability," NASA PT-1224, 1978

${ }^{7}$ Idelchik, I.E., "Handbook of Hydraulic Resistance," $3^{\text {rd }}$ Edition, Section 8-3, Jaico Publishing House, 2008
} 